\title{
El uso de Twitter para analizar el activismo ciudadano: las noticias económicas de los principales periódicos de referencia nacional
}

\author{
María del Mar SORIA IBÁÑEZ \\ marsoria@ucm.es \\ Centro de Estudios Superiores Felipe II (UCM)
}

Recibido: 2 de enero de 2014

Aceptado: 11 de julio de 2014

\section{Resumen}

Twitter se ha convertido en el principal medio de expresión ciudadana y plataforma sobre la que generar una nueva forma de hacer Periodismo. En el caso español, las portadas de referencia nacional y sus contenidos en el microblogging generan un tráfico de conversaciones que es el fiel reflejo de un contexto social y político marcado por la crisis económica. Por ello, este estudio tiene como fin reflejar el grado de participación ciudadana sobre los contenidos informativos en Twitter, partiendo de la cuantificación de sus interacciones o del tono de sus menstajes. El análisis de contenido que presenta este trabajo radiografía el uso que hacen los principales periódicos españoles del nuevo medio social, señala la importancia que otorga la ciudadanía a las noticias vertidas por las secciones de economía y revela cuáles son las publicaciones más activas en el microblogging y, por lo tanto, las más populares.

Palabras clave: Ciudadanía, Twitter, prensa, economía, crisis económica, desempleo.

\section{Using Twitter to discuss citizen activism: the economic news from leading newspapers of national reference}

\begin{abstract}
Twitter has become the primary means of public expression and platform on which to build a new way of doing journalism. In the Spanish case, the national reference and covers its contents on the microblogging conversations generate traffic that is a true reflection of a social and political context marked by the economic crisis. Therefore, this study aims to reflect the degree of citizen participation in the information content on Twitter, based on the quantification of their interactions or their menstajes tone. The content analysis presented in this work radiography their use major Spanish newspapers the new social media, notes the importance it attaches to citizenship expressed by sections of economy news and reveals which are the most active posts on the microblogging and therefore, the most popular.
\end{abstract}

Keywords: Citizenship, Twitter, press, economy, economic crisis, unemployment.

\section{Referencia normalizada}

SORIA IBÁÑEZ, María del Mar (2015): "El uso de Twitter para analizar el activismo ciudadano: las noticias económicas de los principales periódicos de referencia nacional". Estudios sobre el Mensaje Periodístico. Vol. 21, Núm. 1 (enero-junio), págs.: 599-614. Madrid, Servicio de Publicaciones de la Universidad Complutense.

Sumario: 1. Introducción; 1.1. Origen de la participación social 2.0; 1.2. Activistas y alternativas; 1.3. Objetivos; 1.4. El método; 1.4.1. La muestra; 1.4.2. Período de análisis; 1.4.3. Las variables y la categorización; 1.4.4. Sistema de cuantificación. 2. Resultados y conclusiones; 2.1. La relevancia del mensaje; 2.2. El perfil de los ciberactivistas; 2.3. Consideraciones finales. 3. Referencias bibliográficas. 


\section{Introducción}

El trabajo que se presenta tiene como principal objetivo dibujar el perfil activistas online en Twitter y sus principales reivindicaciones ante las noticias que vierten los periódicos de referencia nacional (El País, El Mundo, La Vanguardía, El Périodico, $A B C$, según el EGM) en un contexto económico y social que, en el último trimestre del año 2012, está marcado por la alta tasa de desempleo y las cifras negativas sobre la economía española ${ }^{1}$.

Partimos de la base de que Twitter ha dejado de convertirse en un soporte que fomenta el feedback entre personas que tienen vínculos en común para consolidarse como un medio de opinión pública en el que la ciudadanía plantea debates que pueden orientarse a una mejora del sistema. El medio social se ha convertido en la nueva base del activismo y se impone como alternativa a las protestas ciudadanas a pie de calle.

\subsection{Origen de la participación social 2.0}

La recesión económica ${ }^{2}$ y los cambios políticos y sociales ${ }^{3}$ han contribuido a que el año 2012 haya sido especialmente noticiable por el amplio movimiento social generado en torno a una opinión pública condicionada por una alta tasa de desempleo que llega en el citado período al $26 \%$ de la población activa.

En este contexto, la ciudadanía ha decidido participar de forma más acusada en la vida pública, y las redes sociales han contribuido a generar una nueva forma de comunicación global para hacerse escuchar. De hecho, Ferreras (2011) nos acerca a esta idea partiendo del Movimiento 15-M, la primera gran concentración ciudadana planificada a partir de Internet y cuyo principal canal de comunicación fue el microblogging Twitter. La autora manifiesta que este hecho de mayo de 2011 sentó las bases para crear un nuevo activismo basado en el social media.

El activismo online o ciberactivismo ya forma parte de una realidad social compuesta por una opinión pública que, independientemente de la ideología que profese, pretende participar del debate colectivo a través de la Web 2.0.

Una intensa participación social que no se daba desde comienzos de los años noventa, coincidiendo con un período de recesión económica que dejó también altas cifras de desempleo, aunque reducidas ligeramente hasta el $20 \%$ de la población activa. La situación provocó un aumento de la conflictividad laboral importante entre 1993 y 1996, coincidiendo con la huelga general de 1994 (Jodar, 2006).

1 Según la Encuesta de Población Activa (EPA) correspondiente al cuarto y último trimestre de 2012, el año cerró con una tasa histórica de 26,02\% de desempleo. Ello se traduce en un total de 5,96 millones de personas sin empleo en España. En el caso del colectivo juvenil, la cifra alcanza al 55,12\% del grupo (2,79 millones).

2 La cifra de 5,96 millones de desempleados y el déficit del 6,8\% (confirmado por el propio Gobierno de España en septiembre de 2013) dan buena cuenta de una situación financiera sin precedentes para la economía española.

3 Los datos económicos impulsan el aumento de las manifestaciones ciudadanas. Según datos de las delegaciones del Gobierno en todo el territorio nacional, durante los diez primeros meses de 2012 tuvieron lugar 36.232 reivindicaciones públicas en España, el doble que en 2011. 
Volviendo al presente, las cifras de participación en el social media no dejan lugar a dudas. En España, la consultora Nielsen ha revelado que Twitter cifra ya sus tuiteros en 5,3 millones, aunque Comscore los sitúa en 5,4 millones.

El poder ineludible del microblogging reside en el concepto de instantaneidad y $f e$ edback directo, puesto que el público, a través de un mensaje compuesto por 140 caracteres, puede interactuar con una organización de manera notoria y sin censura (Lovejoy, Waters y Saxton, 2012). Además, y pese a que las cifras de usuarios son menores que en la red social generalista Facebook, lo cierto es que su formato le ha permitido convertirse en la herramienta de la Web 2.0 más habitual para crear ciberinformación y distribuir mensajes relacionados con la actualidad más inmediata (Waters, Tindall y Morton, 2010: 249).

En este contexto, numerosas organizaciones empresariales se han unido a Twitter, conscientes, además, de que el medio les permite generar información fluida en un breve espacio de tiempo, mediante el uso de palabras clave (que en el microblogging evolucionan hasta el denomidano 'hastag' o keywords más populares). De hecho, investigaciones como las desarrolladas por Kwak et al (2010) ya se plantean el grado de alcance del poder mediático que genera Twitter pero, sobre todo, de la posición que ocupa ya como un nuevo medio de comunicación impulsado fundamentalmente por la ciudadanía 2.0.

El activismo online ha dejado de ser un medio puramente unidireccional, como sucedió hace unos años, cuando aún Twitter era un desconocido para los internautas españoles, para consolidares como una plataforma de expresión ciudadana orientada al consenso social en torno a cuestiones que son de interés general (Fernández, 2012).

\subsection{Activistas y alternativas}

Para definir esta figura emergente, la del activista online, podemos seguir a Hopenhayn (2004: 8), que argumenta que es una persona que utiliza un conjunto de técnicas y tecnologías de la comunicación tales como teléfonos móviles, blogs, correo electrónico o redes sociales, organizando, movilizando y sirviendo de inspiración a comunidades online cuyo objetivo es poner en marcha procesos de acción y toma de posición social. Por ello, y gracias a las tecnologías, es posible participar en movilizaciones globales, y de este modo la identidad de los jóvenes se basa en la dinámica propia de la sociedad de la comunicación, que no se encuentra limitada por las distancias geográficas.

Ciberactuar implica, en definitiva, empowering people o, lo que es lo mismo, un proceso mediante el cual la sociedad civil toma parte de la agenda pública mediante el desarrollo de estrategias que persiguen el cambio de la misma o la inclusión de un nuevo tema en el orden del día, a través la difusión de un determinado mensaje y su programación a través del "boca a boca" multiplicado por los medios de comunicación y publicación (De Ugarte, 2007: 85).

Dicho lo anterior, conviene señalar que el término, que ha sido ampliamente estudiado por diversos autores (Torres, 1009; Luengo, 2010; Ferreras, 2011), puede presentar diferentes tipologías que representan el fin que persiguen los usuarios cuando para poner en marcha procesos de actuación y toma de posición social, tal y como destaca Hopenhayn (2004). Así, podríamos hablar de un ciberactivismo político, social, lúdico o comercial. 
No cabe duda de que la actualidad en las redes sociales está marcada por un fuerte activismo político pero también con una importante base social. Es decir, la situación de desempleo generalizado y la pérdida de poder adquisitivo de muchos ciudadanos españoles como consecuencia de una crisis económica que perdura desde el año 2007, pone de manifiesto que, la ciudadanía y, por ende, los usuarios 2.0, están descontentos con la realidad social que viven y lo expresan abiertamente en las redes sociales. De hecho, otras investigaciones sobre activismo online ya han puesto de manifiesto que el germen de las manifestaciones sociales en las redes viene determinado por los perjuicios para la sociedad y para las familias que acarrea la situación de crisis financiera. Así, autores como Gil (2012) o Artigas et al (2012) resaltan en sus investigaciones la importancia de Twitter para generar nuevas corrientes de opinión pública en torno al decaimiento económico que sufre el país. Los autores profundizan en esta cuestión sobre los casos del Movimiento 15-M en España, o en las elecciones venezolanas, respectivamente.

A partir de ello es fundamental hablar de un activismo online de tipo social que está ligado a la participación ciudadana. Así, y partiendo de la tipología de carácter social (Luengo, 2010), podemos tomar como referencia otras obras que ponen el acento sobre la participación ciudadana en la Red con un claro objetivo de cambiar su propia realidad social. Es el caso de la propuesta de Martínez (2011), que determina que las NNTT favorecen que la ciudadanía pueda desplegarse en la vida pública y empoderarse para lograr justicia social.

En este contexto, creemos fundamental reseñar que a presente investigación se orienta a analizar a los activistas online que protagonizan la mayor parte de los mensajes que se generan en los espacios de los medios de comunicación españoles, de forma que podamos categorizar esos contenidos y conocer si sirven para generar consenso y aportar nuevas ideas.

Así, y partiendo de un activismo online social, que revitaliza el papel de la ciudadanía en todos los segmentos, y que pretende generar cambios con sus manifestaciones, analizamos qué ha sucedido en Twitter en los últimos tres meses del año 2012, calificado como el año de la recesión económica, en un país en el que la ciudadanía puede formar parte de nuevas corrientes de opinión y participar del desarrollo de medidas que generen los cambios necesarios para volver a la estabilidad económica y social tan deseada.

\subsection{Objetivos}

Una vez radiografiado el mapa del nuevo activismo online, describimos los fines que nos proponemos en nuestra investigación, bajo la premisa fundamental de conocer el uso de los espacios de los periódicos en Twitter por parte de la ciudadanía, y sus reacciones y propuestas ante informaciones que reflejan la catástrofe de la economía española.

Los fines concretos son:

1. Cuantificar el volumen de interacciones que genera el medio a través de su noticias y analizar la participación del usuario ante las mismas, con el fin de determinar el grado de importancia de la información. 
2. Identificar el perfil de los usuarios que interactúan en los distintos medios de comunicación analizados en Twitter, con el fín dibujar un mapa de públicos de activistas online sobre las noticias de actualidad.

3. Determinar el tono que adquiere el mensaje, de manera que podamos poner de manifiesto las reacciones de la ciudadanía ante informaciones de la realidad social.

\subsection{El método}

Para poder alcanzar los objetivos planteados es necesario aplicar un método que determine la forma y el fondo de los mensajes lanzados por los usuarios que interactúan sobre las noticias publicadas por los periódicos seleccionados a través de Twitter.

Para ello, creemos que el análisis de contenido es la técnica que puede determinar las características del ciberactivismo generado por la opinión pública que presta atención a los medios de comunicación objeto de estudio. Krippendorff (1990:28) realiza una definición muy acertada de esta herramienta metodológica, al definirla como una "técnica de investigación destinada a formular, a partir de ciertos datos, inferencias reproducibles y válidas que pueden aplicarse en su contexto".

Dicho lo anterior, resulta imprescindible diseñar una plantilla de análisis que, a través de criterios cualitativos y cuantitativos, sea el eje vertebrador para alcanzar los fines propuestos diseñados. Proponemos, por tanto, un modelo de estudio aplicado al análisis de los mensajes en Twitter partiendo de los postulados genéricos en torno a las aplicaciones Web 2.0, tal y como defiende la obra de Celaya (2008), y de otros más específicos que se centran de forma concreta en el activismo online.

El diseño de la plantilla de análisis parte de las investigaciones previas de Java et al (2007), Huberman, Romero y Fan Wu (2009) Cha et al (2010) y Kwak et al (2010).

\subsubsection{La muestra}

Para determinar los periódicos objeto de estudio que analizaremos en Twitter nos basamos en el ranking de lectores de medios publicado por el EGM (Abril de 2011 a Marzo de 2012).

De este modo, consideramos oportuno la elección de los que ostentan los cinco primeros puestos, teniendo en cuenta que se trate de publicaciones de carácter nacional y generalista. Por ello, los diarios a estudiar serían los siguientes:

- El País. 1888 lectores por día

- El Mundo. 1.226 lectores por día

- La Vanguardia. 850 lectores por día

- El Periódico. 654 lectores por día

- $\quad A B C .649$ lectores por día

El medio o plataforma que analizamos es el microblogging Twitter, dado que se parte de la premisa de que se ha convertido en le herramienta de activismo online más utilizada por la ciudadanía.

Para afinar aún más el análisis, consideramos relevante la elección de la sección de Economía de las citadas publicaciones sobre las que nos basamos para el estudio, dado que en la misma ofrece una visión muy ilustrativa sobre una crisis económica acom- 
pañada de un desempleo sin precedentes en España. Pero además, nos detenemos, de un modo más concreto en aquellas informaciones que, integradas en la mencionada sección, incluyen los vocablos desempleo y crisis.

\subsubsection{Período de análisis}

Nos planteamos estudiar el cuarto y último trimestre del año 2012, por tratarse de un año especialmente relevante en términos sociales, dado que cifras tales como el desempleo la economía de las familias españolas han dado los peores resultados en la totalidad de la serie histórica de los datos que maneja el Ministerio de Empleo y Seguridad Social.

Dicho esto, los meses de evaluación son:

- $\quad$ Octubre de 2012

- $\quad$ Noviembre de 2012

- $\quad$ Diciembre de 2012

Los periódicos a estudiar son los cinco primeros de carácter generalista incluidos en el último ranking del EGM, y que ya se han mencionado anteriormente.

\subsubsection{Las variables y la categorización}

Para poder obtener los resultados planteados en el proyecto de investigación debemos establecer dos grandes bloques de análisis que integran variables concretas y que serán determinantes para alcanzar los fines propuestos:

- Relevancia del mensaje. Se establecen los siguientes ítems distinguiendo el contenido del medio y el mensaje del público:

- Medio de comunicación. Número de seguidores y volumen de retweets por cada contenido vertido.

- Público. Número de 'tweets' o comentarios por cada noticia, número de seguidores de cada usuario que ciberactúa con un comentario.

- Perfil del usuario. Se estudiarán las siguientes variables: formación académica, ocupación y sexo.

- Tono de las interacciones de los seguidores. Se evaluarán los siguientes puntos: determinar si el tono es negativo o positivo y si hay aportaciones o idas para generar los cambios demandados

\subsubsection{Sistema de cuantificación}

Dada la variedad de variables categorizadas anteriormente expuestas, se establecen los siguientes criterios de cuantificación partiendo de los estudios previos de Java et al (2007), Huberman, Romero y Fan Wu (2008) Cha et al (2010) y Kwak et al (2010).

- La relevancia del mensaje:

La importancia del mensaje se medirá en función del número de interacciones. El análisis ecuacional (correlación de Spearman) nos ayuda a determinar las relaciones entre la relevancia del mensaje y la popularidad e influencia de los seguidores de cada medio. La cuantificación será del siguiente modo: 
a) Medio de comunicación. Mediremos el grado de importancia de las informaciones a partir de las conclusiones previas de Cha et al (2010), que demostraron que la relevancia de un medio en Twitter está relacionada con el alto volumen de seguidores que tenga, el número de retweets a la noticia o el volumen de menciones o la cuantificación de los comentarios a la información que se publica.

b) Público. Mediremos el activismo online de las personas que generan comentarios en las noticias a través de la cuantificación de sus seguidores, pues también queda demostrado (Cha et al, 2010) que cuantos más 'followers' tenga un usuario mayor será su popularidad y por tanto, influencia.

- Perfil del usuario:

Se evaluarán de forma detallada el sexo, la ocupación y la formación académica de los usuarios y se obtendrán resultados que se representarán a través de gráficas y porcentajes.

- El tono de las interacciones:

Se analiza mediante la detección de las siguientes palabras clave:

a) Tono Negativo: reivindicaciones en contra de algún colectivo político, en contra de entidades financieras o protestas no pacíficas

b) Tono Neutro: una postura imparcial

c) Tono Positivo: ¿El usuario aporta soluciones para cambiar la realidad social o es simplemente una protesta?

En este apartado se utilizará como herramienta la distribución Ji Cuadrado de Pearson.

\section{Resultados y conclusiones}

El análisis se ha centrado en la sección de Economía de los periódicos de referencia españoles en su posicionamiento 2.0 en Twitter. Por tanto, partimos de la base de que el grado de interacción de activistas viene determinado por la especificidad del propio espacio (centrado, en ocasiones, en contenidos muy especializados). Sin embargo, el estudio ha arrojado conclusiones muy representativas del ciberactivismo ciudadano en torno a la situación de la economía española y el desempleo, principal preocupación para la sociedad española ${ }^{4}$.

El periódico que registra un liderazgo absoluto en su versión en el microblogging es El País, pues se aprecia un total de 74.155 seguidores 5 . Llama la atención, sin embargo, que publicaciones de gran prestigio e influencia social, como es El Mundo (reflejado en el número de lectores que señala el EGM) cuente solamente con 575 seguidores en Twitter $^{6}$. Una proporcionalidad que refleja un prestigio nada parecido al que cuenta la versión impresa de la publicación, tal y como se puede apreciar en la Tabla 1.

4 Según el barómetro del CIS (Centro de Investigaciones Sociológicas) correspondiente a abril de 2012, el paro es el principal problema para el $81,7 \%$ de la población.

5 El análisis se realizó entre los meses de enero y marzo de 2012, por lo que la cifra responde al citado período, entendiendo que varía posteriormente, aunque no se aprecian cambios significativos en el número señalado.

6 Recordamos que el análisis se ha centrado en las secciones de Economía de cada medio, por lo que el volumen de seguidores es más bajo que en espacio genérico que engloba todos los contenidos informativos que se publican en la edición impresa. 


\begin{tabular}{|c|c|c|}
\hline PUBLICACIÓN & $\begin{array}{c}\mathrm{N}^{\circ} \text { SEGUIDORES EN } \\
\text { TWITTER }\end{array}$ & $\begin{array}{c}\mathrm{N}^{\circ} \text { DE LECTORES VERSIÓN } \\
\text { IMPRESA }\end{array}$ \\
\hline EL PAÍS & 74.155 & 2.859 .281 \\
\hline$A B C$ & 4.171 & 389.069 \\
\hline EL MUNDO & 575 & 1.203 .874 \\
\hline LA VANGUARDIA & 12.666 & 213.961 \\
\hline EL PERÍODICO DE CATALUÑA & 133 & 100.066 \\
\hline
\end{tabular}

La popularidad de cada uno de los medios analizados también es proporcional al volumen de contenidos que arrojan en el espacio virtual. De este modo, el periódico más activo, El País que, como vemos, es el que presenta más ciberactivistas, también es el que ofrece la media de noticias más alta. De hecho, la ratio se sitúa entre 11 y 12 informaciones por mes, teniendo en cuenta que hemos extraído solamente los contenidos que incluían los términos crisis y desempleo. Con ello también podemos determinar que la publicación menos activa en contenidos es El Periódico de Cataluña, pues solamente presenta una media de 7 noticias sobre la situación económica que afecta a la ciudadanía. Ello, como veremos más adelante, repercute en una baja popularidad en el microblogging acompañada, como no podía ser de otro modo, por la escasa participación de la ciudadanía. Algo que no sucede en El País, donde el número de noticias llega acompañado de un importante ciberactivismo.

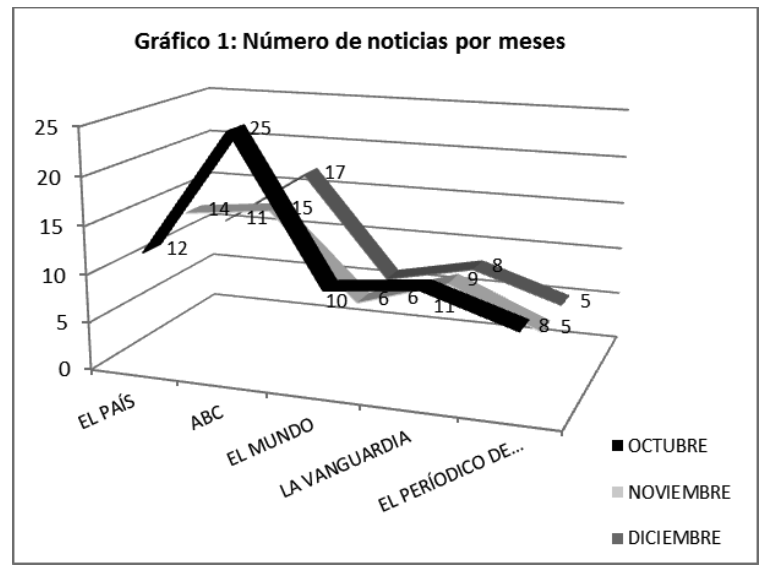

Cuanta más información genere el medio, mayor será la participación de la ciudadanía. El País vuelve a liderar este criterio, pues presenta una media de 5,8 interacciones (comentarios a las noticias publicadas) de usuarios por noticia analizada, aunque el mes de octubre es el que refleja un mayor número de comentarios, coincidiendo con noticias de gran envergadura como es el arranque del curso político o la subida interanual del paro en un $10,84 \%$. En noviembre y diciembre la mayor parte de las informaciones publicadas giran en torno al balance anual de todos los sectores de la economía española, aunque la participación es menos notable, pensamos que 
debido a que no se proporcionan, durante los dos últimos meses del año, datos relevantes sobre el desempleo anual ${ }^{7}$.

Por su parte, El Periódico de Cataluña y El Mundo son las dos publicaciones que presentan un menor número de interacciones de los usuarios, teniendo en cuenta que es de 0,8 y de 4,3 comentarios de media por cada información vertida, respectivamente. Poca participación especialmente sorprendente en el caso de El Mundo, que ocupa el segundo lugar en el ranking del EGM de prensa generalista.

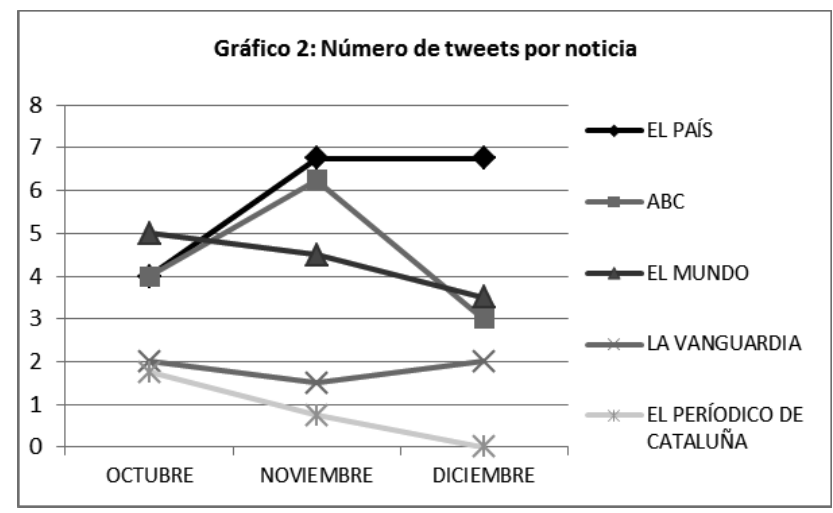

\subsection{La relevancia del mensaje}

Por otro lado, al analizar el número de retweets que se generan por cada noticia, nos encontramos con que el diario $\mathrm{ABC}$ ostenta en este punto el protagonismo indiscutible. Pese a que solamente cuenta con una media de 2,5 tweets a las noticias que genera en Twitter, los usuarios que interaccionan y divulgan esos contenidos más que en ningún otro medio. Algo que se aprecia en el número de retweets que generan esos comentarios en $\mathrm{ABC}$ y que, durante tres meses, alcanzan la media de 21,3. Una ratio muy por encima de que la presentan El Mundo y El Periódico de Cataluña, que durante el período de análisis solamente consigue una persona de media preste atención a sus contenidos, dándole mayor difusión a través del retweet.

Dicho esto, no cabe duda de que, si atendemos al criterio señalado, y pese a que el periódico $\mathrm{ABC}$ cuenta con menor número de seguidores e incluso aunque la mayoría de ellos no genera opinión pública, sí que son más influyentes que los que presentan el resto de medios, en tanto en cuanto su contribución a la divulgación de contenidos es más prolífera que en los demás periódicos de referencia. Le siguen en este punto, por orden de importancia, El País (con una horquilla entre 3 y 7), La Vanguardia (24,8) y El Periódico de Cataluña y El Mundo (1-2).

7 Sin embargo, a comienzos del año 2013 se publica un dato muy notorio sobre el mapa del paro en España, que hubiera generado, sin duda, una gran controversia en la Red. La EPA (Encuesta de Población Activa) reveló que el paro creció en 2012 en 691.700 personas con respecto al año anterior 


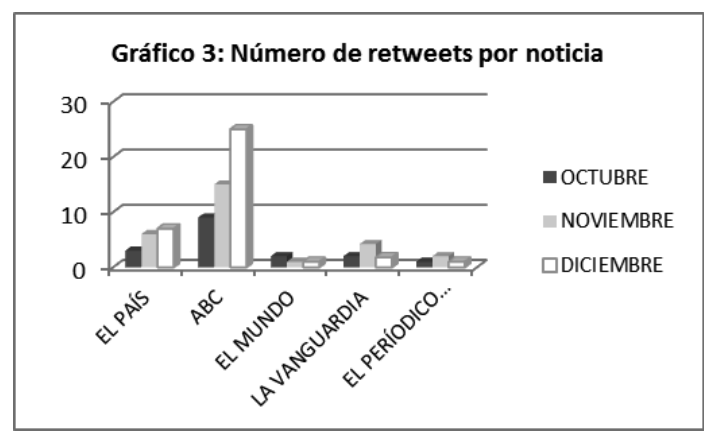

Vemos, en términos generales, que hay una correlación de moderada a alta entre la popularidad e influencia de un determinado medio de comunicación, aspectos medidos a través de su número de seguidores, y la importancia que otorgan los ciberactivistas a las noticias vertidas (la cual está baremada a través del número de retweets que generan dichos ciberactivistas) ${ }^{8}$. De este modo, el grado de importancia de las informaciones las determinan los propios usuarios, pues cuantos más seguidores haya más difusión se creará, y más relevancia adquirirá el mensaje para la opinión pública de Twitter.

\subsection{El perfil de los ciberactivistas}

Los resultados arrojados por el análisis de los rasgos que definen a los activistas de cada medio ponen de manifiesto que, de nuevo, El País destaca en este punto. Ello significa que los ciudadanos que comentan las informaciones vertidas por el periódico tienen de media 391 seguidores, partiendo del período de estudio analizado. Le sigue La Vanguardia (306), ABC (213), El Periódico de Cataluña (14) y El Mundo (11). En este punto cabe recordar las demostraciones de Cha et al (2010), que llegaron a la conclusión de que cuanto más número de seguidores tenga un activista online, mayor será su popularidad y, por tanto, influencia en el resto de usuarios. Por ello, y volviendo a nuestros resultados, sin duda El País es el medio que cuenta con los ciberactivistas más populares e influyentes.

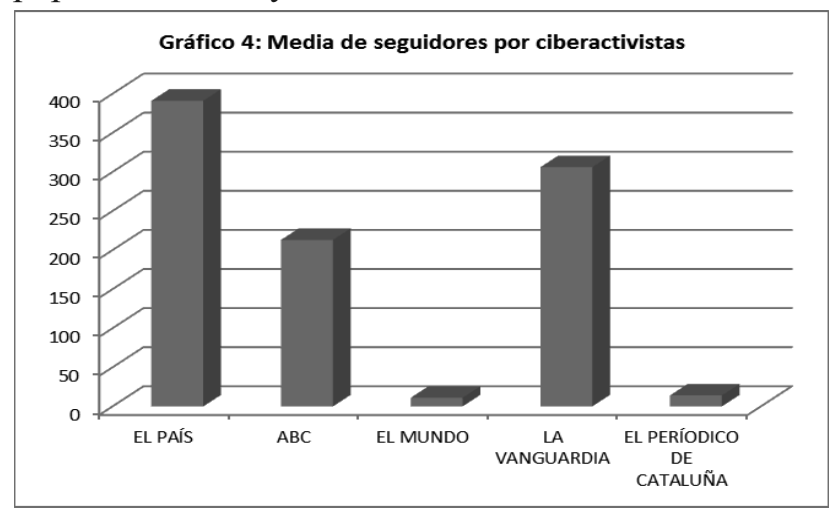

${ }^{8}$ Correlación de rangos de Spearman. Rs $=05105$ 
En lo que se refiere al sexo mayoritario de las personas que debaten los contenidos informativos, prevalece la participación masculina en todos los medios analizados. Es significativa la representación en el periódico $A B C$, publicación en la que el $57 \%$ de las personas que opinan sobre la información son hombres. Los datos más pobres los encontramos en El Mundo y El Periódico de Cataluña, en los que la ausencia de participación nos impide determinar el sexo de los activistas online, ya que hay una mayoría notoria que expresa anonimato en este sentido.

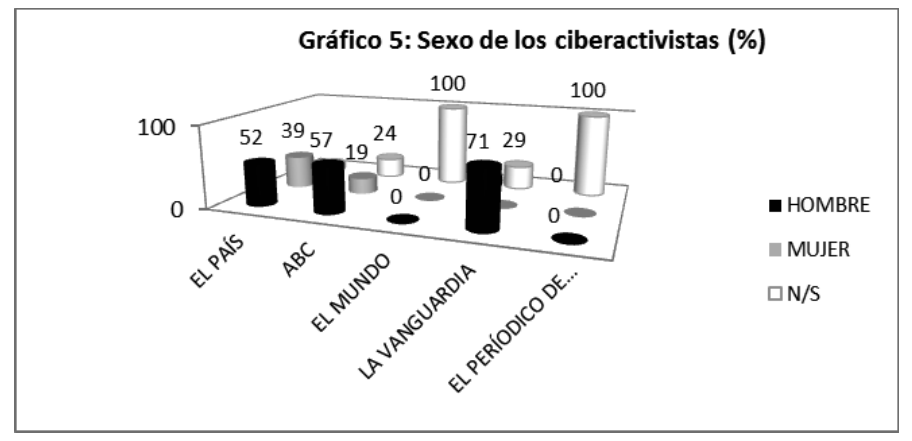

No cabe duda de que hay una mayoría masculina (condicionada, pensamos, por cuestiones de desigualdad de género en los distintos estratos sociales y sectores profesionales y la consecuente brecha digital) y de que los usuarios que poseen estudios universitarios lo incluyen en su ficha personal. La Vanguardia es la publicación que registra un mayor volumen de ciberactivistas que afirman poseer estudios universitarios (43\%), mientras que las versiones en Twitter de la sección de economía de El Periódico de Cataluña y El Mundo ofrecen datos muy pobres al respecto, dada que la escasa participación en estos medios obstaculiza el análisis del criterio señalado.

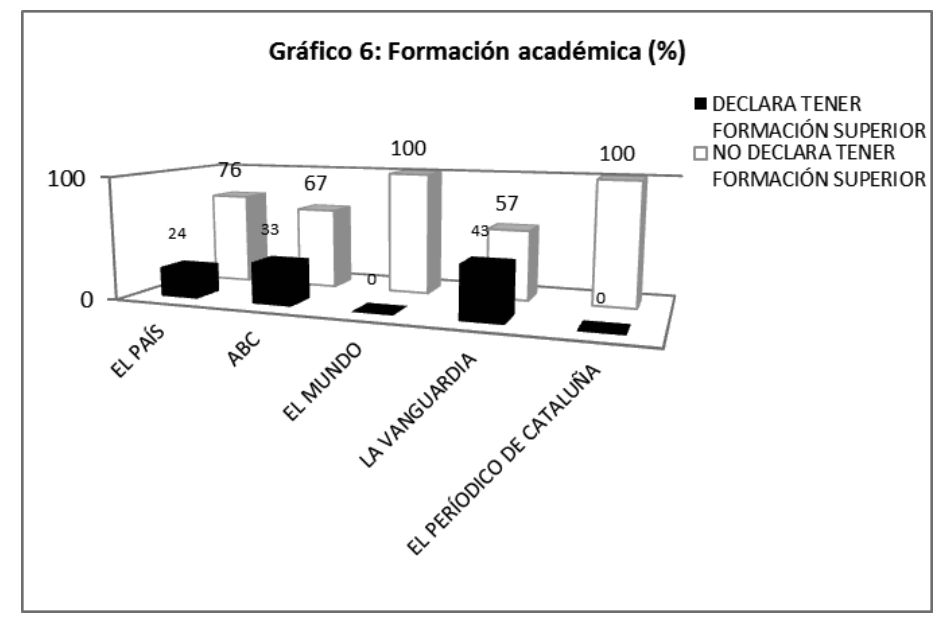


¿Cuál es la profesión mayoritaria de esos usuarios que comentan las noticias? Lo cierto es que la heterogeneidad de los usuarios nos impide hablar taxativamente de porcentajes representativos. Sin embargo, insistimos, pese a la variedad de profesiones observadas (partiendo de los titulados universitarios), hemos comprobado que los perfiles más habituales son los profesionales procedentes de la abogacía (más numerosos en El País), economistas (más numerosos en El País) personal sanitario (más numeroso en $\mathrm{ABC}$ ) y periodistas.

Por último, y sobre el tono que adquieren los comentarios de los usuarios, creemos que lo más relevante es que no se detecta ningún comentario positivo sobre las noticias vertidas por los medios seleccionados para este estudio. Ello viene determinado por la realidad de que la mayor parte de las informaciones son de carácter negativo. Los malos datos del paro o del déficit público avalan el malestar de la ciudadanía, que no duda en poner de manifiesto su opinión, generalmente mostrando un pesimismo latente sobre la situación económica que atraviesa España en el último trimestre de 2012.

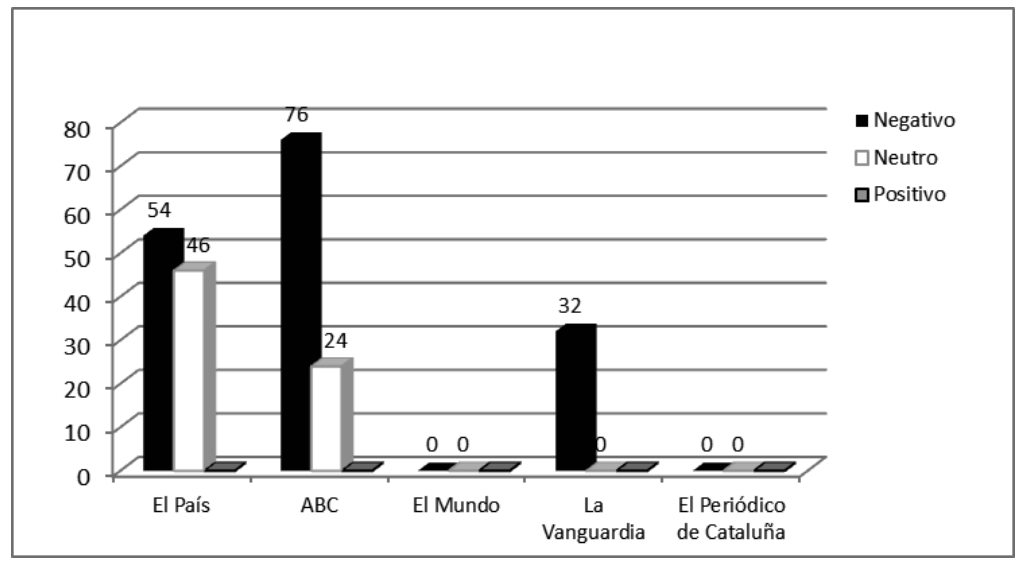

\subsection{Consideraciones finales}

Nuestro análisis pone de manifiesto que el medio más activo en Twitter es, con diferencia, el periódico El País. Se alza como la publicación que emite un mayor volumen de contenidos informativos de índole económica, y ello provoca también que sea el periódico que recibe un mayor y eficaz feedback por parte de la ciudadanía. De hecho, la media de contenidos diarios se sitúa en 6,75. Y es en este punto, recordemos, en el que la mayoría de los usuarios expresa libremente su opinión, si bien es cierto que existe una tendencia muy extendida a la crítica hacia los poderes públicos.

Por el contrario, $A B C$ se convierte en la publicación que obtiene un mayor número de retweets sobre sus publicaciones, hasta llegar a la media de 25 por noticia durante el mes de diciembre. En este caso no se trata de una interacción en la que la palabra tome el protagonismo, pero sí se trata de una acción que contribuye a difundir el contenido noticioso del medio a través del soporte social, al tiempo que los usuarios lo comparten con sus amistades en Twitter. 
No obstante, recordemos que el establecimiento de opiniones y comentarios ante las noticias de actualidad aportan un gran valor al discurso del medio, puesto que contribuye a generar diversidad de ideas y, por su puesto, a establecer otros enfoques sobre el mensaje que el medio no puede plantear por tener que ceñirse a su función informativa.

Sobre el volumen de seguidores, el periódico El País vuelve a liderar este criterio. Se convierte en la publicación que alcanza los 74.155 fans (en su sección económica). Ello provoca también que cuente con personas afines que gozan a su vez de mayor popularidad en el microblogging. Un criterio que se evidencia a partir del siguiente dato: los seguidores de El País tienen una media de 391 amistades por usuario, y se convierte así en la publicación que ostenta el mayor ratio en esta línea. Ello le proporciona mayor popularidad en la Red.

Sobre la formación académica de los seguidores de las publicaciones objeto de estudio, y analizando uno a uno los usuarios, comprobamos que existen dos tipos bien distintos: los que se proclaman con estudios universitarios y los que sencillamente no aportan información. Así, nos encontramos con que la mayoría de los fans de las publicaciones mantiene la privacidad de ese dato, entendemos que para salvaguardar su vida profesional y proteger su intimidad.

Ello provoca también que en El Mundo y en El Periódico de Cataluña los usuarios no manifiesten su sexo. De hecho, hay una tendencia muy extendida a incluir fotografías de perfil que contienen ilustraciones abstractas, así como el uso de seudónimos que protegen la identidad de los usuarios.

Por último, y sobre el enfoque que adquieren los mensajes de la ciudadanía, hemos comprobado que prevalece el tono negativo. Los usuarios se muestran pesimistas y reclaman medidas para poner solución al desempleo creciente en España. Pero además, $\mathrm{y}$ de forma sorprendente, nos encontramos con que no existen comentarios de los ciudadanos ante las noticias de El Mundo y El Periódico de Cataluña. Algo que puede tener su razonamiento en el hecho de que las secciones de Economía de ambos periódicos en Twitter tengan vetada la participación de los usuarios.

Por otro lado, y en términos generales, hemos de concluir que la participación ciudadana es media y que podría ser mucho más fluída. Se ha tenido en cuenta que el análisis ha partido de una sección especializada como es la de Economía y que sesga al público de forma inevitable. Sin embargo, hemos querido partir de la misma porque muestra con objetividad datos económicos, en algunos casos demoledores, que hacen que hayamos podido conocer al usuario y a sus principales manifestaciones.

Así, y aunque de una forma moderada, el mapa ciudadano se muestra desilusionado y pesimista ante un contexto económico y social que tiene un precedente similar en la crisis de 1.993.

El periódico El País resulta el medio más interactivo y en el que se concentra el mayor volumen de ciberactivismo. Algo que viene determinado por su activo papel en Twitter y por una retroalimentación eficaz.

Creemos, no obstante, que, a tenor de los resultados, las secciones de Economía de los principales periódicos de referencia nacional España pueden y deben tener un mayor y eficaz espacio en Twitter, pues se convierte en el medio más fluido y directo 
para que el usuario aporte y manifieste sus inquietudes a partir de la divulgación de informes cuantitativos que, en muchos casos, se dirigen solamente a un público especializado. Twitter ofrece la posibilidad de democratizar la sección de Economía, y la gratuidad e instantaneidad del medio invita a esta evolución.

\section{Referencias bibliográficas}

ARTIGAS, Daniela; MUÑOZ, Ángel; LUENGO, Francisco; CHOURIO, Xandre; y FERNÁNDEZ, Andrea (2012): "Caracterizando las elecciones venezolanas a través de Twitter: Caso \#26S". Disertaciones, Anuario Electrónico de Estudios en Comunicación Social, vol. 5, núm. 1, pp. 57-76. Venezuela, Universidad de Los Andes.

BLANCHET, Alain; GHIGLIONE, Rodolphe; y MASSONNAT, Jean (1989): Técnicas de investigación en Ciencias Sociales: datos, observación, entrevista, cuestionario. Madrid, Narcea.

CASTELLS, Manuel (1997): La era de la información. Economía, Sociedad y Cultura. Madrid, Alianza Editorial.

CASTILLO, Antonio (2010): "La comunicación de los lobbies en Internet: el ciberactivismo de los Think Tanks". Icono 14, vol. 8, núm. 1, pp. 193-206. Madrid, Asociación Científica Icono 14. Universidad Complutense de Madrid.

CELAYA, Javier (2008): La empresa en la Web 2.0. Barcelona, Gestión 2000.

CHA, Meeyoung; HADDADI, Hamed; BENEVENUTO, Fabricio; \& GUMMANDI, Krishna P. (2010): "Measuring user influence in Twitter: The Million Follower Fallacy". Fourth International AAAI Conference on Weblogs and Social Media. Washington, George Washington.

DE UGARTE, David (2007): El poder de las redes. Manual ilustrado para personas, colectivos y empresas abocados al ciberactivismo. Bilbao, Grupo Cooperativo de las Indias.

DUNLAP, Joanna and LOWENTHAL, Patrick (2009): "Tweeting the night away: Using Twitter to enhance social presence". European Journal of Information Systems, vol. 24, num. 2, pp. 1-8. Birmingham, The OR Society.

FERNÁNDEZ, Juan Sebastián (2012): “Ciberactivismo: conceptualización, hipótesis y medida". ARBOR, Ciencia, Pensamiento y Cultura, vol. 188, núm. 756, pp. 631639. Madrid, Consejo Superior de Investigaciones Científicas.

FERRERAS, Eva (2011): "Redes sociales y cambio social: el movimiento 15-M y su evolución en Twitter". Telos: Cuadernos de comunicación e innovación, 89, pp. 6173. Madrid, Fundación Telefónica.

GONZÁLEZ SAN RUPERTO, Marta (2007): "Ciberactivismo social en las ONG: los casos de Amnistía Internacional, Intermón Oxfam y Greenpeace”, pp. 128-134. En: WALZER, Alejandra; GARCÍA, Marcial; y RODRÍGUEZ, Juan Carlos. (coords., 2007): Comunicación alternativa, ciudadanía y cultura. Madrid, Editorial 
Edipo. Esta obra recoge los contenidos presentados a Foro Universitario de Comunicación. Jornadas.

HONNEYCUTT, Courtenay and HERRING, Susan C. (2009): "Beyond microblogging: Conversation and collaboration via Twitter", in Proceedings of the FortySecond Hawai'i International Conference on System Sciences (HICSS-42), pp. 1-10. USA, CA, Los Alamitos, IEEE Computer Society. Hawai, Universidad de Hawai.

HOPENHAYN, Martin (2004): "Participación juvenil y política pública: un modelo para armar". Ponencia presentada en el I Congresso da Associação Latino Americana de População, ALAP, realizado en Caxambú- MG - Brasil, 18- 20 de septiembre de 2004.

HUBERMAN, Bernardo; ROMERO, Daniel; \& WU, Fang (2009): "Social networks that matter: Twitter under the microscope". First Monday, Pee-Reviewed Journal on the Internet, vol. 14, num. 1, pp. 1-9. Bridgman, University of Illioinis.

JAVA, Akshay; SONG, Xiaodan; FININ, Tim; \& TSENG, Belle (2007): "Why we twitter: understanding microbbloing usage and communities". The Philosophical Review, num. 73, pp. 39-460. USA, Universidad de Maryland.

KWAK, Haewoon; LEE, Changhyun; PARK, Hosung; \& MOON, Sue (2010): "What is Twitter? A social network or a news media?". Proceedings of the 19th International World Wide Web (WWW) Conference, April 26-30, 2010, Raleigh NC (USA), pp. 591-600. USA, NY.

KRIPPENDORFF, Klaus (1990): Metodología de análisis de contenido. Teoría y práctica. Barcelona, Paidós Ibérica.

LOVEJOY, Kristen; WATERS, Richard; SAXTON, Gregory (2012): "Engaging stakeholders through Twitter: How nonprofit organizations are getting more out of 140 characters or less". Public Relations Review, vol. 38, issue 2, pp. 313-318. Amsterdam, Elsevier.

LUENGO, Gemma (2010): "La movilización social en Internet. Eventos organizados a través de la red: ¿Fenómeno lúdico o ciberactivismo?”, en IV Congreso Online del Observatorio para la Cibersociedad. Madrid, Observatorio para la Cibersociedad.

MARTÍNEZ NICOLÁS, Manuel (2011): "De la brecha digital a la brecha cívica. Acceso a las tecnologías de la comunicación y participación ciudadana en la vida pública". Telos, Cuadernos de Comunicación e Innovación, núm. 86. Madrid, Fundación Telefónica.

NIELSEN (2012): Spain is digital. Tendencias y consumo online en España. Madrid, The Nielsen Company.

NOGUERA, José Manuel (2010): "Redes sociales como paradigma periodístico. Medios españoles en Facebook". Revista Latina de Comunicación Social, vol. 65, pp. 176-186. La Laguna (Tenerife), Universidad de La Laguna. 
PARK Alexander and PAROUBEK, Patrick (2010): "Twitter as a corpus for sentiment analysis and opinion mining". Language Resources Evaluation Conference, pp. 1-10. Malta, ERLA.

SAKAKI, Takeshi; OKAZAKI, Makoto; \& MATSUO, Yutaka (2010): "Earthquake shakes Twitter users: real-time event detection by social sensors", en Proceedings of the 19th International World Wide Web (WWW) Conference, April 26-30, 2010, Raleigh NC (USA).

SORIA, María del Mar (2010): "El ciberactivismo, nuevo modelo de relaciones públicas en las ONG". Icono 14, pp. 288-302. Madrid, Asociación Científica Icono 14, Universidad Complutense de Madrid.

TORRES, Luis César (2009): "Ciberprotestas y consecuencias políticas: reflexiones sobre el caso de Internet necesario en Méjico". Razón y Palabra, núm. 70. Méjico, Instituto de Estudios Superiores de Monterrey. En: http://www.razonypalabra.org.mx/TORRES_REVISADO.pdf

WATERS, Richard; TINDALL, Natalie.; \& MORTON, Timothy. (2010): "Media Catching and the Journalist-Public Relations Practitioner Relationship: How Social Media are Changing the practice of media relations". Journal of Public Relations, vol. 22, Issue 3, pp. 241-264. New York, Taylor\&Francis. 